\title{
L'innovation territoriale et l'innovation sociale au prisme du discours participatif. Prendre part et prendre une part à l'innovation des territoires.
}

\author{
Territorial Innovation and Social Innovation in regard to a participatory
}

approach.

\author{
Antonella Tufano ${ }^{1}$ \\ ${ }^{1}$ Ecole Nationale supérieure d'Architecture de Paris La Villette, antonella.tufano@paris-lavillette.archi.fr
}

\begin{abstract}
RÉSUMÉ. L'innovation est aujourd'hui la modalité de développement des projets à l'échelle de la ville et des territoires. L'innovation des territoires devient ainsi un déploiement techno-structurel qui poursuit la manière classique de " planifier ». Depuis plus de vingt ans, sous l'influence d'intellectuels, cette planification a dû aussi se questionner au regard des questions sociales et intégrer une forme d'ouverture grâce à la participation. Dans ce but, l'innovation sociale et l'innovation territoriale ont été associées sans questionner la capacité de chaque acteur (public, privé, semipublic) à se reformer sans renier son rôle classique. L'analyse proposée invite à regarder si et comment les démarches participatives facilitent l'implémentation de l'innovation dans les territoires.

ABSTRACT. Both city design and territorial planning seem to be subjected to the imperative of innovation. Territorial innovation becomes a technique which pursues the classic way of "planning". For more than twenty years, under the influence of intellectuals, this way of planning has also had to deal with social questions. In this paper, we explore, through a historic approach, the overlap of social and territorial innovation in regard to participatory approaches and the agencies which could implement them.

MOTS-CLÉS. Participation, innovation territoriale, innovation sociale, design social, échelle.

KEYWORDS. Participatory approach, territorial innovation, social innovation, social design, scaling.
\end{abstract}

\section{Introduction}

Les pratiques de l'urbanisme, de l'architecture et de l'aménagement sont impactées par l'impératif de l'innovation qui se résume ou par l'innovation territoriale, entendue comme l'innovation technique qui concerne les infrastructures (visibles ou moins visibles, comme les smart-grids) ou par l'implantation d'activités économiques. Cette innovation est supposée s'accompagner d'une innovation sociale et «citoyenne» qui peut rendre plus «performants» les territoires, sans préciser les critères qui permettent d'identifier sur quoi porte cette amélioration et quels sont les acteurs de cette transformation. En contexte français, ce croisement entre innovation territoriale et innovation sociale, qui a fait l'objet de nombreuses analyses dans d'autres pays, se heurte à des formes politiques et organisationnelles solidement enracinées dans une hiérarchie décisionnelle et des interfaces institutionnelles aux compétences bien délimitées. La révision de certains plans d'urbanisme offre un exemple actuel : cette compétence gérée autrefois exclusivement par les communes doit s'adapter à la mise en place d'un échelon intercommunal (Métropoles, Communautés Urbaines...) qui comporte une innovation territoriale importante, une coordination «supra-territoriale» dont les modalités de fonctionnement n'ont pas été pensées au préalable. La mise en place de méthodologies d'innovation étudiées dans des contextes autres que ceux spécifiques à la gestion urbaine suscite la méfiance des professionnels de l'aménagement qui considèrent les « spécialistes de l'innovation » (les chercheurs,les managers...) comme des « communicants » qui proposent le « Graal du "faire mieux avec moins"، 1 .

\footnotetext{
${ }^{1}$ Plusieurs articles de la Gazette des Communes se sont faits l'écho des questions posées par le rapport d'Akim Oral, L'innovation au pouvoir. Rapport pour le SGMAP CRED, 2015 ; cf. Sabine Blanc, « L'innovation territoriale sur un fil d'équilibriste », La Gazette des Communes, 17/6/2015 : http://www.lagazettedescommunes.com/370157/linnovation-territoriale-sur-un-fil-dequilibriste/ 
De son coté, l'innovation sociale, lorsqu'elle se résume à l'activité de quelques associations ou groupes de "non-experts", se renferme dans un discours "contre» les institutions qui est trop contextuel et n'arrive pas à faire émerger des politiques consensuelles de transformation des territoires. ${ }^{2}$ De surcroît, en France, la construction de l'intérêt général à l'échelle de l'Etat a un poids décisionnel qui s'accompagne d'une « satisfaction de l'opinion » qui stigmatise les positions non institutionnelles : un exemple emblématique et récent, est le cas paradoxal de l'aéroport Notre Dame Des Landes. ${ }^{3} \mathrm{Au}$ bout d'années de contestations militantes, un referendum a été organisé et la voix majoritaire (exprimée, par ailleurs, par la presque totalité des élus de terrain) a porté à un choix d'aménagement « classique », proposé par les collectivités territoriales. Quelques mois plus tard, par la décision de l'Etat, ce choix a été renversé et la position autrefois radicale a été de facto légitimée de manière très verticale. Ce changement de position qui aurait pu être accueilli comme une violation de la voix populaire a été reçu très favorablement par l'opinion.

Cet épisode bien connu exprime une complexité française qui ne peut pas se réduire aux débats qui traversent depuis quelques années la sphère publique et sont souvent réduits à l'opposition entre un discours bottom up porté par une « minorité » et un discours top down dominant, porté lui aussi par une « minorité », mais qui dispose, elle, d'un réel pouvoir décisionnel. Dans cette opposition quelque peu forcée, depuis les années 1990, a fait son apparition une notion de «valeur » qui nuance le dogme monolithique de «l'intérêt général » et le transforme en un «bien commun » dont les contours restent à préciser. Les dispositifs institutionnels ont alors affiché une volonté d'ouverture et les pratiques non institutionnelles qui ont contribué à faire monter le niveau de connaissance technique d'un public «non expert» ont obtenu une place visible dans les débats. Mais, ce parcours n'est pas rectiligne et la correspondance directe entre innovation territoriale et innovation sociale se réduit à un « discours » (Mollard, 2012) affirmant que les formes participatives et leurs dispositifs d'empowerment conduisent nécessairement à des développements bénéfiques pour les territoires ainsi qu'une plus grande connaissance techno- scientifique (environnement, techniques d'aménagement, choix énergétiques...) des «non-experts ». Certaines initiatives ont ainsi proposé de "redesigner » les politiques publiques pour construire des interfaces d'expressions adaptées à ce savoir émergent de la communauté.

Les paradoxes soulignés plus haut invitent à questionner la généalogie et les formes que prennent les deux termes - innovation sociale ou «citoyenne» et innovation territoriale - ainsi que les manières de transférer l'une à l'autre.

Plus particulièrement :

- le terme « capacitation citoyenne » (empowerment) est souvent évoqué dans les discours de l'innovation sociale ; or, ce terme implique une capacitation de chaque citoyen (Rappaport, 1984) alors qu'en réalité l'expression publique passe par un « groupe », dit minoritaire déterminant la mise en place d'interfaces institutionnelles qui lui correspondent (mais qui négligent les individus) (Deslauriers, 2007)

- la modalité pour transférer les pratiques et le discours de l'innovation sociale dans les territoires est complexe ; en effet, le critère facilitant le transfert semblerait la notion de proximité (Boschma, 2004 ; Torre, 2009), mais, dans des études récentes, il semblerait que les milieux innovateurs ne se comportent jamais de manière linéaire (par exemple, le numérique a remis en discussion la notion même de proximité et sa spatialité)

- Pour établir un lien entre des échelles différentes, tantôt spatiales, tantôt socio-culturelles, a émergé le concept de «scalabilité» qui surmonterait la fracture (Gras, 1993) entre l'échelle

\footnotetext{
${ }^{2}$ Toujours dans la presse professionnelle, des articles récents soulignent la difficulté à appliquer les propositions des associations ; cf. Adeline Farge, « Des innovations associatives au service des territoires encore trop peu reconnues », La Gazette des Communes, 8/12/2017 : http://www.lagazettedescommunes.com/539616/des-innovations-associatives-au-service-des-territoires-encore-trop-peureconnues/

${ }^{3}$ Une synthèse critique est offerte par Hérodote, Géopolitique de l'Environnement, n ${ }^{\circ}$ 165, 2017/2.

(c) 2018 ISTE OpenScience - Published by ISTE Ltd. London, UK - openscience.fr 
anthropologique de l'individu et les technostructures institutionnelles qui rationalisent les discours ; la "scalabilité» pourrait être la notion et la démarche qui permet d'identifier la «bonne échelle » pour trouver une interface d'échange et d'implémentation des innovations sociales sur le territoire.

Pour illustrer ces questions, nous aborderons d'abord la transformation de la pratique de l'aménagement en une théorie discursive; ensuite, comment le discours de l'innovation sociale, s'insinuant dans cette brèche, a pris le pas sur les démarches d'aménagement classiques et a imposé un tournant participatif ; nous aborderons, pour terminer, certaines limites spatiales à l'application des discours qui seront observées à travers le prisme de la proximité et de la «scalabilité».

\section{Le territoire et son innovation}

La transformation de la pratique de l'aménagement en une pratique discursive se développe dans les années 1980, à la suite de la crise de 1973 qui remet en discussion un modèle de développement urbain s'appuyant sur la croissance économique et industrielle des territoires. Ce discours contestataire met à mal le système de validation des décisions par échelons, allant du Plan, dicté par l'Etat, aux Communes, en passant par les administrations déconcentrées (les Directions Régionales et Départementales de l'Equipement, par exemple). Mises en place après la Guerre, ces institutions portaient un regard global sur le développement du territoire et la décision «descendait 》 du niveau ministériel au territoire en connaissant des chaines de validation successives. Ce système fortement centralisé a permis de mettre en place des plans très ambitieux comme les Villes Nouvelles ou, plus récemment, les Opérations d'Intérêt National (OIN) comme celle du plateau de Saclay (Merlin, 2007 ; Alvergne, Taulelle, 2002 ; Caro, Dard, Daumas 2002).

Dans les années 1980, un important mouvement de reconnaissance de la place du citoyen dans les dispositifs décisionnels, porte à une ouverture des dispositifs, à l'ajout de l'échelon régional (qui a en charge les schémas de cohérence territoriale) et surtout à une nécessaire «médiatisation» des opérations d'aménagement (Saint-Pierre, 2002). Ce climat contribue à creuser la séparation importante entre la revendication d'une pratique innovante de l'aménagement portée par des associations et l' innovation institutionnelle et sociale qui permettrait d'implémenter ces expérimentations : les expériences récentes et en plein essor des collectifs d'architectes (Chiappero, 2017) illustrent cette difficulté à transformer une action ponctuelle en une démarche innovante.

D'une part, la parole citoyenne, invoquée par les architectes ou les aménageurs, dans le cas des innovations urbaines, semble trop limitée par le cadre législatif : par exemple, l'importation en France des «jardins participatifs» inspirés par la guerrilla gardening américaine ${ }^{4}$ a donné lieu à des manifestations intéressantes, mais encadrées par les Villes et leurs Services ${ }^{5}$. D'autre part, l'urgence de certaines questions territoriales relevant de l'écologie (urbaine, sociale etc.) demandent une rapidité et technicité de la réponse qui ne peuvent pas s'accommoder des temporalités longues que les négociations demandent. Ainsi, la démocratie citoyenne à l'œuvre dans l'urbain a suscité des critiques (comme dans le cas récent de Réinventons nos places à Paris); les Collectifs d'Architectes en charge de l'étude de l'aménagement ayant un statut associatif leur mission reste une «préconisation » qui ne relève pas la contrainte technique liée à la gestion d'un espace public ou son évolutivité (Fleury, Wuest, 2016).

Pourtant, ces solutions sont utilisées comme l'étendard d'une volonté municipale d'ouverture à la co-conception et sont utilisées pour communiquer tant sur des supports classiques (médias, presse écrite...) que dans la sphère numérique; les habitants -ceux réellement associés aux activités

\footnotetext{
${ }^{4}$ Un livre retrace la naissance et l'évolution de ce mouvement : Reynolds R., On Guerrilla Gardening, Bloomsbury, 2008

${ }^{5}$ Par exemple, à Paris, la gestion de l'ensemble des jardins participatifs est confiée à La Main verte qui est intégrée au Service de 
participatives et ceux qui en prennent connaissance dans les médias- ont ainsi l'impression de participer à un choix collectif (Mollard, 2012).

Le passage par le discours aurait pu permettre de créer une interface d'échange entre la pratique technique (et la parole codée) des responsables de l'aménagement et l'expression d'un groupe d'usagers de la ville ; cette rencontre aurait pu aussi permettre aux « non experts » de s'émanciper par l'acquisition et la pratique d'un langage sinon technique au moins « sachant» au regard du lexique urbain.

Il en a été autrement. Sans rentrer dans l'explicitation des évolutions de la théorie de l'aménagement, de l'urbanisme ou de la pensée du territoire (Ascher, 2010 ; Levy, 2013 ; Levy, Lussault, 2000 ; Lussault, 2007), il convient de souligner la disjonction croissante entre le territoire et ses politiques d'aménagement qui empruntent un modèle de développement urbain -sinon inspiré- au moins pensé en relation à l'expansion économique et industrielle de la ville (Perulli, 2009 ; Pinson, 2009).

La première modernité de l'urbanisme (fin XIXe siècle- années 1930) a permis de constituer les outils qui ont été appliqués dans les années 1950-1970 pour donner forme au modèle du développement de cet urbanisme industriel (Choay, 1979). Dans les années 1980, lorsque le terme « développement durable » apparaît, il porte «l'espérance d'un tournant environnemental » (Brundtland, 1987) ; son ouverture sur le social intervient dans les années 1990 (Le Gales, 1995), avec la question de la gouvernance territoriale, et, enfin, à partir des années 2000, ce terme déjà ancien s'approprie les approches culturelles (Florida, 2000 ; Morisset, Noppen, 2005) pour inciter à un nouveau type de "croissance durable ». A la suite de ces mutations, les institutions intègrent les démarches innovantes (territoriales, institutionnelles, sociales...) dans toute opération d'aménagement qui touche le milieu (Berque, 2000) : s'établit ainsi un discours sur les milieux innovateurs (Tabariès, 2007) qui a été peu entendu par les professionnels de l'aménagement, en charge de mettre en place les dispositifs « concrets » du développement territorial (les collectivités publiques ou les SEM, Sociétés d'Economie Mixte, auxquelles les collectivités délèguent les aspects opérationnels). L'impasse discursive dans laquelle les Etats (avec des politiques aussi diverses que celle des Etats Unis ou de la France) se sont retrouvés a laissé se renforcer le discours techniciste, d'un côté, et une opposition sociologique de l'autre côté.

En France, comme le montrent de nombreuses études historiques, les corps d' ingénieurs ont investi depuis longtemps les politiques territoriales, urbaines et d'aménagement, forts de leurs compétences techno-scientifiques, consubstantielles d'un pouvoir technocratique et institutionnel. Les deux volets de la légitimité des ingénieurs se renforçant mutuellement (technocratie et expertocratie), la disjonction avec les savoirs des autres experts du territoire -architecte, urbaniste et, parfois, les géographes et sociologues de l'urbain- n'a fait que s'accroitre et ceux derniers sont restés à l'extérieur du monde opérationnel.

Le terme innovation et, tout particulièrement le terme innovation territoriale, reste ainsi cantonné à l'usage presqu'exclusif de ces corps techniques (Raineau, 2014 ; Forest, Hamdouch, 2015) et suscite l'indifférence ou même le rejet de ceux qui l'associent à un développement économique étanche aux impacts sociaux et culturels que cela implique dans les territoires. Comme le souligne Fleury (2018), le défi posé par l'architecture et l'urbanisme à la théorie de l'innovation n'est pas facilement surmontable. En effet, s'il existe bien un cadre normatif pour tous les domaines de développement des innovations (Gaglio, 2011), il est plus contraignant dans le cas de l'architecture et de l'urbanisme où il y a autant de prototypes que de projets et autant de clients institutionnels que d'identités territoriales ; de ce fait, même si chaque terrain ou chaque bâtiment demande une innovation, l'unicité sur laquelle repose l'expérimentation ne permet pas d'en construire un cadre normatif facilitant son transfert à

\footnotetext{
${ }^{6}$ Il est possible de souligner l'apparition concomitante dès la fin du XIXe siècle du terme urbanisme par Cerda à Barcelone, avec les contraintes techniques liées à cette activité, et la critique de la ville moderne faite par Simmel. 
d'autres «terrains ». Selon cette position chaque projet-création ne dépasse jamais le cadre de l'invention (ou du prototype) pour devenir une innovation, c'est à dire une « invention appropriée par la société » (Caron, 2010; Desjeux, 2017). Ce constat, qui fait suite à de nombreuses tentatives d'analyse conduites dans le champ de l'architecture et de l'urbain en France, depuis les années 1980 (Boudon, 1990 ; Conan, 1990 ; Prost, 2015), mérite une nuance. D’abord, il faut séparer l'innovation architecturale et l'innovation urbaine, car elles relèvent d'histoires, de cultures et méthodes différentes. L'innovation architecturale peut donner lieu à des développements industriels et des transferts culturels qui s'apparentent à l'innovation technologique (Callon, 1996; Picon, 2010; Terrin, 2015). L'innovation urbaine mobilise des temps plus longs de débat, d'acceptation partielle d'une proposition de projet et la validation nécessaire des usagers qui ne peut pas se mesurer mais simplement « apprécier » par un feed-back assez aléatoire (Tsiomis, 2007). De surcroît, la difficulté à mesurer l'impact de chaque cas de projet, ne permet pas de vérifier si l'expérience est transposable et s'il est possible de transformer l'action sur le terrain en un modèle.

Ensuite, malgré l'affichage de la volonté à construire une connaissance interdisciplinaire dès la phase de formation des techniciens de l'aménagement (Vinck, 1999), l'absence de lieux où l'enseignement est entièrement transversal (réf.) et nourri par les débats détermine une méfiance à l'égard des discours portés par « les autres » professionnels et, encore plus, envers l'apport du débat public dès qu'il assume des formes conflictuelles.

Enfin, la transformation de la pratique codée de l'aménagement en un sujet ouvert à la discussion peut, certes, donner lieu à l'empowerment du citoyen mais aussi se transformer en exercice rhétorique de domination des experts (politiques et techniciens). Ainsi, au fur et à mesure que les notions abstraites liées à la technique s'incarnent dans des territoires elles génèrent des " figures urbaines » technologiques qui brident le débat public en raison de la technicité requise pour comprendre leur fonctionnement : un exemple évident est la notion de smart associée à l'innovation urbaine, telle qu'elle est présentée, par exemple, par le Plan Urbanisme Construction Architecture du Ministère de l'Environnement (PUCA). La smart city a été réduite au développement de réseaux de transfert technologiques, à la gestion des centres de traitement des données qui y sont associés et aux connexions possibles avec les infrastructures territoriales qui régissent la gestion des flux matériels (transports) et immatériels (énergie, communication...) (Salat, 2011). Cette séparation entre le territoire et sa pensée infrastructurelle n'est pas seulement le fait d'un corps d'ingénieurs spécialistes, mais aussi de groupes privés qui interviennent dans la gestion urbaine via des Partenariats Public Privé (les PPP) qui leur accordent un contrôle global sur la res publica. La ville d'Issy-les-Moulineaux ${ }^{7}$ est l'illustration de ce développement accompagné par le privé. L'image de "ville innovante » choisi par la ville d'Issy comme un outil de communication, de branding territorial, ne correspond guère aux études urbaines conduites par les chercheurs en sciences humaines et sociales qui n'arrivent pas à identifier quel type d'innovation sociale ou territoriale a été produite par le choix du développement des technologies numériques dans la ville. Par ailleurs, le partenariat avec le privé impose une discrétion absolue dans la diffusion de l'information car il faut respecter le secret industriel et le droit des brevets nécessaires à l'exploitation économique de l'innovation.

Un exemple similaire est l'association entre les big datas et les politiques de construction territoriale impulsées et conduites par ces partenariats soutenus par le privé (Scherrer, 2010) ; ce discours «politico-administratif» peu ouvert à la critique intra-disciplinaire empêche également d'affirmer d'autres postures. Ainsi, les expériences d'empowerment citoyen par le numérique ${ }^{8}$, qui fabriquent une intelligence individuelle de la ville et pourraient donner lieu à du «médiactivisme» (Cardon, Granjon, 2013), font figure d'alternative peu crédible et le discours politique se concentre sur la solution technique de la « fracture numérique ».

\footnotetext{
${ }^{7}$ En raison des brevets et autres formes de protection, les seules informations et feed-backs de cette expérience sont fournis par les collectivités impliquées dans le développement de la Ville (CCI, Région, CDC) et les opérateurs privés (Bouygues).

${ }^{8}$ Par exemple, le groupe des Citoyens capteurs qui conduit des relevés de pollution urbaine à Paris qui montrent des résultats très différents de ceux des institutions (Natureparif). 
La disparition d'un marquage net entre les compétences publiques et celles du privé se traduit en un discours à double visage, un pharmacon - alertant à la fois sur les dangers et les remèdes- qui est mobilisé dans les projet selon une géométrie variable. Le remplacement progressif d'une autorité publique gérant l' «intérêt général » et la délégation au privé ouvre un débat politique aussi dans les théories de l'urbanisme qui se déclarent innovantes : est-il possible de séparer le «bon acteur privé » qui se soucie du «bien commun», de l'acteur privé intéressé qui pourrait profiter « commercialement » du bénéfice offert par la gestion ouverte de l'urbain ? La polémique actuelle sur les démarches de «réinvention» urbaine de la Ville de Paris en est l'exemple". Les signataires d'un article, paru dans Libération le 6 février 2018, condamnent la décision de la Ville de confier "au privé » la construction de passerelles piétonnes sur la Seine. Le motif est le caractère naturel exceptionnel de la Seine, un «bien commun » des parisiens, un élément fondamental dans le paysage urbain de la capitale classé à l'Unesco. Or, de manière étonnante, parmi les signataires, on trouve des architectes et des théoriciens qui ont défendu la co-construction urbaine et les nouvelles formes de partenariat avec le privé. Comment distinguer donc la «bonne innovation» de la co-production urbaine, de la «mauvaise»? Les signataires, en utilisant l'argument du paysage naturel du fleuve, comme un cadre fixe à respecter, ne font-ils pas appel à un « naturalisme nostalgique » (Ferrari, Assennato, 2016) qui engendre le commerce lucratif des touristes et des fortunes étrangères qui viennent profiter à Paris d'une image pittoresque artificiellement figée ? Il n'est ici pas question de trancher, mais de souligner les risques d'interprétation qui se manifestent dans le transfert du discours à l'aménagement opérationnel. L'innovation, sociale et territoriale, serait donc un pharmacon, et chaque facette mériterait d'être étudiée en relation aux impacts complexes des innovations urbaines.

L'absence d'une critique constructive semble donc le fait d'un manque d'échange interdisciplinaire, entre les opérateurs publics, les acteurs privés, industriels, et les chercheurs (sociologues de l'urbain, théoriciens de l'urbanisme, comme le montrent certaines des recherches (Arab, 2014) qui regardent le terrain de manière pertinente, mais ne tentent pas l'expérience du croisement méthodologique au-dela des domaines proches (création, conception, technique). Conduite dans des milieux universitaires ultraspécialisés (CRAUP, 2018), l'innovation territoriale revendiquée dans les recherches des architectes et ingénieurs, se cantonne à l'observation de micro-phénomènes -comme la "créativité dans l'urbain » (Kunzmann, 2005 ; Boren, Young, 2013) - dont la validité en tant que facteur d'innovation sociale à l'échelle d'une ville reste à démontrer (Arab, Vivant, 2018). Les « entrepreneurs de méthodes » salués dans les expérimentations conduites au nom de l'innovation territoriale par les collectivités sont souvent des artistes, des paysagistes-jardiniers ou des designers qui traduisent les échanges d'acteurs et/ou d'experts en micro-projets censés fonctionner comme des déclencheurs de la participation. Il s'agit donc de figures de la «médiation», souvent semi-institutionnelles, ayant des contrats d'assistance à la maîtrise d'ouvrage, qui n'échappent pas aux pièges tendus par une « institutionnalisation » de la participation citoyenne que les sociologues observent, parfois de manière très critique, depuis une vingtaine d'années.

\section{La transformation des pratiques d'aménagement en discours}

En retraçant un historique de l'innovation, Benoit Godin (2015), souligne que le mot appartient au registre politique depuis l'Antiquité, mais que son apparition dans les discours politiques se fait en France au XIXe siècle. Son impact réel sur les décisions de terrain intervient, lui, bien plus tard, dans les années 1980 de manière concomitante à la critique de la théorie urbaine de la planification. En France, la «théorie» de la planification urbaine se résume à un ensemble de discours institutionnels portant sur des aspects techniques et règlementaires, de politiques publiques, parfois de justifications socio-économiques, mais elle n'a pas fait l'objet d'une théorisation générale comme c'est le cas dans

\footnotetext{
${ }^{9}$ Voir à ce propos l'article: « La Seine n'est pas à vendre », Libération, 06/02/2018 / http://www.liberation.fr/debats/2018/02/06/laseine-n-est-pas-a-vendre_1627859 
d'autres pays. ${ }^{10}$ La particularité de la politique urbaine, en France, est de demeurer une « action » qui se déroule en trois temps : études, règlements, mise en œuvre opérationnelle; cette priorité donnée à l'acte de «faire » conduit d'une part à une efficacité de réalisation qui « autolégitime » la pratique, mais, d'autre part, exclue la question de l'innovation territoriale (et plus particulièrement son volet social), en raison de son caractère interdisciplinaire et discursif. Dès les années 1960 (Lefebvre, 1968 ; Lefebvre, 1973) l'opérationnalité de l'aménagement est questionnée au regard de la valeur politique de l'espace ; confrontée au discours politique (Harvey, 1973 ; Harvey, 2000 ; Garnier, 2001), en tant que vecteur de légitimation égal au savoir techno-scientifique de l'aménagement; ces critiques émises par des théoriciens «externes» à la fabrique politique de l'urbanisme insistent sur le fait que la planification doit s'ouvrir à la confrontation des discours, faute de quoi elle risque de devenir le lieu de fabrication d'une « injustice spatiale » (Busquet, 2007). Comme le note Hamel (1997), la théorie de la planification est peu étudiée en tant que « discours de légitimation » car elle est prise entre l'exigence de reconnaissance scientifique de la discipline (Forester, 1992 ; Barattucci, 2006) et l'incompréhension des praticiens de l'urbain (Hemmens, Stiffel, 1980) : cette affirmation est vraie dans tous les contextes nationaux, mais plus encore en France car chaque acteur -de l'urbanisme, de l'aménagement et du projet urbain- se concentre sur la réalisation et s'ouvre peu à l'échange verbal avec des spécialistes de l'urbain issus des champs théoriques. C'est qu'en fait cette théorie, notamment lorsqu'elle veut évoquer la prise en compte du local, comme dans le texte de l'urbaniste italien Magnaghi (1990 et 2014), se trouve prise entre une double intention: d'une part, se poser en tant que dispositif opérationnel permettant d'engager une démarche territoriale à l'échelle locale, la bio-région, avec l'appui du politique, et, d'autre part, l'ambition opposée qui consisterait à construire un « modèle scientifique de référence » capable de « théoriser » le territoire (Choay, 1979 ; Choay, Merlin, 2000) et s'incarner à chaque fois dans une forme hyper-territoriale différente et innovante (Lussault, 2017). Ainsi, toute «émergence» urbaine (non seulement les lieux consolidati (selon l'expression de Bernardo Secchi), comme le centre-ville, mais aussi les lieux touristiques, les fab-labs, les tiers lieux, les campements informels...) devient une manifestation visible d'une évolution socio-politique des lieux (Soja, 2000 ; Lussault, 2013). Il s'agit donc, comme le suggère Kaesler (1996), de revoir la différence wébérienne entre ordre des faits et ordre des valeurs qui seraient ici superposés et non opposés, ce qui se traduirait par la superposition entre l'innovation territoriale et l'innovation sociale.

La rupture qui intervient dans les années 1980 (Fishman, 1980) est le marqueur de la volonté de réunir, d'une part, une théorie de la planification ontologiquement attachée à une vision moderne de progrès et de croissance et, d'autre part, un discours sur la ville en train de se faire ${ }^{11}$ avec le retour à des valeurs autres, immatérielles, qui ne sont pas mesurables en termes de progrès et croissance, mais en tant que valeurs symboliques (Claval, 1999). Ce discours a été construit en milieu anglo-saxon avec beaucoup de cohérence (entre les objectifs politiques et les dispositifs urbains) tandis qu'ailleurs il est resté cantonné aux expérimentations de certains pays (scandinaves, baltiques, alémaniques) dont les conditions politiques permettaient l'émergence des formes participatives (Pinson, 2009).

En milieu américain et canadien, cette construction d'un « discours de la théorie de la planification » qui s'oppose à la pratique du projet territorial est portée par un courant communicationnel (Milroy, 1991) qui se donne comme finalité de faire émerger la parole du citoyen et lui permettre d'exprimer sa subjectivité ; la légitimation de cette parole permet de construire un espace public habermassien où la dimension symbolique prévaut sur le fonctionnalisme. D'ailleurs, la reprise des textes du sociologue Georg Simmel ${ }^{12}$ à cette époque (Tiryakian, 1992) correspond à un regain d'intérêt pour l'écologie urbaine de l'école sociologique de Chicago (Jacobs, 1961). Il s'agit donc d'un moment charnière car il permet de faire émerger une figure «polémique » de l'urbain qui trouvera son écho dans plusieurs pays, mais dont l'application concrète se heurtera aux pratiques et dispositifs institutionnels en place.

\footnotetext{
${ }^{10}$ Par exemple, la foundation de l'urbanisme attribuée à Cerda, à Barcelone, se fonde sur un "acte de planification”, mais elle deviant immédiatement une "théorie" affichée par l'auteur dans unouvrage. Cette demarche existe également dans d'autres pays, notamment l'Italie où les travaux de Secchi, Campos ou Cervellati ont été traduit et diffusés (Françoise Choay).

${ }^{11}$ Il s'agit d'une collection des éditions Parenthèses où publient les chercheurs : Desvismes, Godiet, Tapie, Veltz...

${ }^{12}$ Notamment les textes contenus dans Simmel G., Philosophie de la modernité, Paris, Payot, 1989

(c) 2018 ISTE OpenScience - Published by ISTE Ltd. London, UK - openscience.fr 
Réduites à la communication, les pratiques de planification adoptent les valeurs de cette médiatisation et se donnent comme objectif de respecter et mettre en valeur le bien public et le bien commun. Ainsi : " les recherches sur l'espace public, de circulation ou de communication, sont indissociables de la redéfinition, dans les démocraties contemporaines, des notions de bien public et de bien commun. L'espace public est, en effet, le premier des biens publics, qu'on le conçoive comme visibilité mutuelle, espace de rencontre ou mise à disposition de chacun d'une intention » (Isaac Joseph, 1992).

La centration sur la figure de l'individu permet de réinventer des formes autres de solidarité ou d'accepter la notion d'indifférence urbaine dans sa complexité (Leontidou, 1996). Comme le résume Hamel : «Cela a d'abord conduit à dépasser les conceptions abstraites de la citoyenneté et de la démocratie, afin de tenir compte d'éléments subjectifs ou existentiels ouverts sur la quotidienneté et l'urbanité » (Hamel, 2004) qui constituent le lit de la réflexion conduite par Henri Lefebvre sur le « droit à la ville » (Henri Lefebvre, 1968).

Cette réouverture au « citoyen » (Ellin 1996; Hamel et Poitras 1994) a conduit à la nécessité de se faire entendre et d'entendre les discours marginalisés. Concentrée auparavant sur les aspects normatifs et d'aménagement (droit de l'urbanisme ; génie urbain ; art et/ou projet urbain), la théorie urbaine a dû construire un « discours » permettant de créer une interface de communication entre des acteurs aux langages, compétences et intentions radicalement différents : ce cadre initialement réservé aux ingénieurs, juristes et urbanistes, s'est ouvert aux sociologues et aux anthropologues qui y ont introduit de «nouveaux acteurs» (associations, collectifs d'architectes de l'éphémère, praticiens du «jardin urbain »....). Dans ces nouveaux cadres, la seule interface possible est celle de la parole ; militante, engagée, ludique, cette irruption de la parole a conduit à une mutation inédite des études urbaines qui doivent «rendre accessibles» les compétences spécifiques (Tapie, 2000) et construire une vision pluraliste de l'aménagement, en passant de ce fait d'une esthétique à une éthique de l'urbain (Blanc, 2015).

Aux Etats-Unis, le discours du New Urbanism (Calthorpe, 1993 ; Calthorpe, Fulton, 2001), du Social Design (Sommer, 1983) ou de la Post-metropolis (Soja, 2000) tentèrent d'expliquer des phénomènes spatiaux par les conditions socio-économiques. L'éclatement de la centralité urbaine, la référence au «vernaculaire» urbain, correspond ainsi à une forme de création spontanée de la ville : Zevi parlera à ce propos de « dialectes» de projet (Zevi, 1996).

Pour Forester (1989), la planification coïncide avec le processus politique et va dans le sens d'une démocratie participative, où la communication revêt autant d'importance que la matérialité de la ville (selon la théorie habermassienne de l'agir communicationnel). Au tournant des années 1990-2000, ces visions et discours urbains ont rencontré et épousé les revendications des militants qui voulaient prendre part spécifiquement à ce qu'ils considéraient comme des «bien communs » et le premier d'entre eux, l'environnement (Hardin, 1968 ; Ostrom, 1990) ${ }^{13}$ La force du discours communicationnel associé à la notion de «bien commun » investit les questions d'environnement, qui reposaient jusqu'à lors essentiellement sur un socle scientifique (Déleage, 1991; Bourg 2014 ; Bourg, 2015). Pour le courant post-moderne de la théorie urbaine, la connaissance technoscientifique existe, et elle se visibilise dans le discours dominant du scientifique/technicien associé au pouvoir (comme le développent Gottdiener, en 1993, Healey, en 1996, et Topalov en 2015), mais elle doit être soumise à la même critique qu'on porte envers le concept de "progrès »; de cette posture émerge un discours écologique qui montre une compétence technique et discursive des acteurs dits « minoritaires » qui, en réalité, sont issus des même milieux techniques que les aménageurs (Cans, 2006).

La communication est également mise en œuvre dans le cadre d'une pensée postmoderne du territoire, où elle sert à rendre visibles les tensions et à susciter le débat sur une refondation

\footnotetext{
${ }^{13}$ Pour un aperçu de ce débat, voir l'article d'Hervé Le Crosnier dans le Monde Diplomatique : https://blog.mondediplo.net/2012-06-

15-Elinor-Ostrom-ou-la-reinvention-des-biens-communs

(c) 2018 ISTE OpenScience - Published by ISTE Ltd. London, UK - openscience.fr

Page $\mid 8$
} 
démocratique, mais elle n'a aucune visée ou valeur opératoire (Mouffe, 1992). Dans ce dernier cas, la nature discursive de la théorie urbaine s'adresse aux planificateurs (Leontidou, 1996) qui sont chargés de mettre en place la transformation démocratique et la seule "agitation des formes langagières " constitue l'aboutissement de ces démarches.

Nous remarquerons ici que la mutation de paradigme interprétatif et de «théorie urbaine» des années 1980 réside dans le changement positionnel de deux entités : jusque dans les années 1980 (la modernité sociologique), c'est la forme spatiale qui prime pour l'interprétation des mutations urbaines et territoriales. A partir du postmodernisme, c'est la structure politique, sociale et économique qui est privilégiée pour comprendre la «mutation spatiale». Ce changement de point de vue, entre territoire et méta-territoire (social, économique...), s'opère par la mise en œuvre d'un nouveau discours de légitimation qui, depuis la fin des années 1980, s'impose progressivement. La seule manière de capter ce changement est de mesurer la place accordée aux discours discordants, ceux de la minorité qui s'oppose au discours de l'expert technicien (Ellin, 1996) et permet de construire une nouvelle figure de la réalité urbaine (Ghorra-Gobin, 2015).

Cette transformation de la ville en une figure urbaine et celle des théories urbaines en des « discours » maniables politiquement ouvre donc la voie à l'apparition de l'impératif participatif. En France, le conflit de l'Alma-Gare à Roubaix (Hatzfeld, 1986), démarré en 1966, avec la décision de la municipalité socialiste de raser intégralement ce quartier populaire, devient un laboratoire urbain qui voit l'émergence d'acteurs -ni institutionnels, ni techniques- qui se regroupent autour d'un lieu novateur, l'Atelier Populaire d'Urbanisme. Porteur de la voix de ces classes populaires qui devraient être logées ailleurs, il devient un outil de contestation qui va débattre avec les représentants politiques et les aménageurs pour essayer d'infléchir leur politique d'aménagement. A partir de 1973-1974, débute donc, ici, la première « concertation urbaine » française. Cette discussion conflictuelle finit par l'emporter, en 1983, grâce au soutien d'un maire inscrit dans le courant réformiste du Parti Socialiste. A la sociologue et politiste Hélène Hatzfeld de remarquer que, dans cette expérience à Roubaix, le conflit est devenu une forme d'échange qui peut permettre d'arriver à des compromis et que ce dispositif, peu utilisé jusqu'à là, a acquis une légitimité pour acter des décisions de transformation urbaine. Ces médiations se transformeront dans les démarches participatives, collaborative, partagées qui seront intégrées aux nouvelles théories et politiques de l'urbain dans les années 1990.

\section{Le discours sur la participation : la légitimation de quoi ?}

Les analyses critiques des modèles de la démocratie représentative (Anselme et alii, 2001 ; Rui, Gourgues, Topçu 2013) montrent la montée en puissance d'une rhétorique participative qui permet de créer une interface entre le langage institutionnel et normatif et celui du non-expert (les habitants, les usagers, les associations, bref la "société civile»). La caractéristique de cette interface est d'être également une démarche d'acquisition, partage et transmission de savoirs (Schön, 1995 ; Stenberg, 2012) qui n'hésite pas à avoir recours à un discours médiatique ou communicationnel. De la médiation au médiatique, le pas est bref, comme le montre la multiplication d'acteurs d'interface sortis du monde médiatique et s'offrant comme «coach » des politiques dans les discussions publiques (Linossier, 2012; Rey-Valette, Syndia, 2012). Au milieu des années 1980, par exemple, les promoteurs de l'empowerment écologique aux Etats Unis, étaient des experts qui opéraient un lien entre l'opportunisme économique et la demande sociale et n'hésitaient pas à se transformer en figures des médias $^{14}$.

De ce mélange, naît une idée de la participation où les revendications politiques et l'expertise sont transmises avec un langage médiatique qui semble démocratiser la connaissance de l'expert (Arnstein, 1969). La place de l'habitant/individu rentre ici dans une négociation où les limites de l'acceptabilité

\footnotetext{
${ }^{14}$ Ian MacHarg et son émission sur la planète et ses relations avec Nixon ; cela donnera lieu à un événement très populaire aux EtatsUnis, l'Earth Day 
individuelle coïncident souvent avec celles de la « communauté ». Sans rentrer dans les détails de la théorie proposée par Sherry Arnstein, il convient de rappeler qu'elle proposait d'évaluer le poids que les politiques accordaient à la voix des non-experts à travers l'analyse des dispositifs de participation mis en place. Et, en synthétisant les expériences conduites sur plus de trente ans, le rapport de la DIV (Délégation Interministérielle à la Ville, 2012) a tiré la conclusion que la délégation de pouvoir, ou le contrôle citoyen, n'étaient encore que des horizons théoriques (DIV, 2012). Ce constat sévère, venant d'un des acteurs les plus importants et volontaires, en France, en matière d'innovation politique des territoires, mérite donc d'être analysé.

En effet, dans le transfert des discours de politique urbaine en France, et notamment au regard des politiques participatives dans l'urbain (Gauthier, Baqué, 2013), la valeur contextuelle de ce qui est « acquis pour les habitants » prime sur l'acquisition de compétences et connaissances par les nonexperts. Cela engendre une tension forte, d'autant plus que la notion " procédurale » anglo-saxonne de «bien commun» qui préside à l'évaluation des acquis définit sa valeur en fonction de l'interaction, tandis que «l'intérêt général» des pratiques de l'aménagement repose sur la reconnaissance d'une valeur substantialiste, propre à l'Etat ou à la puissance publique (Blanchot, Padioleau 2003 ; Padioleau, 2000).

De surcroît, le discours bottom up des démarches américaines est issu (et fut porté par) une « voix communautaire ", tandis qu'en France, la diffusion de ce modèle alternatif s'est fait de manière descendante, du milieu universitaire (par exemple à l'université de Vincennes, en 1968, dont l'expérience est abordé dans le livre Les sociologues contre l'Etat, 1986) vers les quartiers identifiés comme le lieu possible d'un conflit. L'introduction française de ces démarches est due essentiellement à la DIV et ses «Entretiens de la Ville», qui se sont déroulés de 1991 à 1993, ont donné un rôle central à des intellectuels comme Jean Baudrillard, Alain Touraine et d'autres universitaires cette présence d' intellectuels dans le débat participatif se prolonge jusqu'au rapport intitulé Ça ne se fera pas sans nous (Bacqué, Mechmache, 2013) qui est une commande de l'Etat.

Toujours dans le sillage de ce discours centré «bottom up» paradoxalement promu par un discours «top down», on constate que l'importation «à la française» du community planning, se réduit à l'action de quelques experts indépendant qui proposent des séances de discussion dont l'objectif est de « mesurer l'acceptabilité » d'une décision y compris en introduisant une notion de « conflictualité nécessaire » (Mansbridge et alii, 2010), bien plus que les capacités à participer en amont aux choix urbains. En effet, il semblerait que l'acquisition de compétences dans l'urbain - dispositifs juridiques ; compréhension du coût d'un aménagement et des techniques de mise en oeuvre ; compétence dans l'évaluation des critères esthétiques d'un projet...- n'est jamais une des priorités des promoteurs de ces démarches participatives dans l'urbain, à la différence des démarches de sensibilisation aux risques naturels (ou, plus en général, des démarches initiées pour défendre des environnements). Comme le remarquent Barbier et Larrue (2011), c'est à partir du moment où la préoccupation environnementale devient un "problème à résoudre », c'est-à-dire le moment où elle sort de son langage scientifique et devient un sujet politique et de démocratie, que l'environnement rentre dans le débat public. Il devient alors un enjeu et l'opérateur d'une sorte de démocratisation du savoir scientifique. Tatenhove et Leroy (2002) notent que très rapidement s'opère une superposition entre green discontentment (Vandana Shiva, 1991), participation et une volonté de modernisation politique qui détermine la naissance du cadre législatif permettant aux dispositifs d'interface et interaction de se mettre en place (ClaeysMekdade, 2006 ; Blatrix, 2000). La particularité de cette participation à la gestion de la Nature est qu'elle mobilise des enjeux économiques encore plus importants que l'urbain et -en même temps- est investie d'une image qui va au-delà de la satisfaction contextuelle; relevant de la valeur intrinsèque de la ressource naturelle, elle touche toute la société, ici et ailleurs, maintenant et dans le futur (Flipo, 2007 ; Audier, 2017).

A défaut de construire une explication complexe, ce débat autour de la «société écologique » a fait l'objet d'un cadrage réglementaire très contraint presque pour éviter qu'elle déborde des cadres 
institutionnels fixés (Mermet, Berlan Darqué, 2009). La modernisation des institutions s'est ainsi incarnée en une rationalisation de l'action publique, qui classe: participation, consultation ou négociation, mettant en scène les différents stades de la dynamique «conflit -coopération». Ce mouvement de rationalisation s'est accéléré avec l'entrée dans «l'âge du risque» (Beck, 1986) et les dispositifs de protection de l'environnement (surtout de l'environnement industriel) ont constitué et constituent le premier cadre contractualisé d'un échange action publique/expertise/citoyenneté sur le risque. ${ }^{15}$ Le seul objectif de cette construction apparente du débat serait -du point de vue praxéologique- de proposer seulement un ordre de l'action : instrumentale (cadre construit par les institutions pour créer les conditions d'une acceptation mutuelle); substantielle (négociation entre le savoir experts et les valeurs invoquées par les autres parties prenantes); normative (« démocratisation de la démocratie » et participation à la Res Publica).

Par ce cadrage, l'administration centrale a favorisé l'émergence de figures institutionnalisées (Beuret, Pennanguer, Tartarin, 2006) qui ont un rôle d'accompagnement; le monde associatif est de ce fait devenu un acteur reconnu et -comme le note Ballan (2011)- cela donne lieu à une limitation de la parole. En cela, les logiques de participation bien rationnalisées sont la modalité pour arriver à une paix socio-environnementale, permettant d'éviter à la fois l'expression d'un individu et, d'autre part, une réelle logique de partage et démocratisation du savoir scientifique. A cet égard, dès 2009, Cécile Blatrix souligne qu'un terme semble absent dans le discours français : le «design participatif», développé au Etats Unis et en Angleterre, qui évalue la qualité des dispositifs et cadres participatifs et propose des guide-lines d'une action pensée avec « chaque habitant».

De manière plus explicite, le cadre anglo-saxon donne comme objectifs de la négociation : d'éduquer le public, d'incorporer des valeurs et intérêts du public dans le processus de prise de décision, d'améliorer la qualité intrinsèque des décisions et de renforcer la confiance dans les institutions, car ces capacitations pourraient réduire les conflits et justifier le temps et le coût consacrés à la participation (cost-effectiveness) (Badré, de Fleurieu, Juffé, 2010). En France, au contraire, les objectifs demeurent flous et le cadre normatif semble institué pour canaliser et freiner le débat démocratique. Il est ainsi possible de constater que dans les débats à fort enjeu économique (Allain, 2010), comme la gestion de l'eau ou les déchets, la concertation a souvent légitimé le discours dominant.

L'inversion de la question s'impose donc : l'environnement a-t-il besoin de la concertation et, plus spécifiquement (Rumpala, 2008) : «le développement durable appelle-t-il davantage de démocratie? Quand le développement durable rencontre la gouvernance, ne s'agirait-il pas d'un programme de gouvernement pour le développement durable?».

Un triple besoin de clarification serait donc nécessaire pour construire les bases d'une innovation sociale et écologique :

1-la relation avec l'institutionnel, qui parfois intervient sous des vestes différentes (EDF qui se diversifie entre sa présence en tant qu'acteur institutionnel, sa présence en tant que financeur d'associations et sa présence en tant que commanditaires d'études et recherche d'innovation territoriale qui légitiment ses demandes);

\footnotetext{
${ }^{15}$ En France, dans la décennie 1990, le débat est cadré institutionnellement par la Commission Nationale du Débat Public de la loi Barnier, en 1995, puis l'article 7 de la Charte de l'environnement, en 2005, et, enfin, après les propositions du Grenelle divers types normatifs de consultation ont été mis en place. D'une part, les commissions publiques qui sont consultatives et largement portées par les acteurs institutionnels, ne font que prolonger les actions publiques classiques. Les non-experts sont surtout les acteurs associatifs qui finissent par disparaitre des négociations assez rapidement car cela demande un engagement personnel trop important (Larrue). D'autre part, la Commission Nationale du Débat Public utilise un modèle emprunté à des expériences étrangères, mais, par exemple, la méthode des jurys, inspirée par des universitaires et qui devrait constituer une spécificité française, a le défaut d'assigner des rôles ; même si la parole peut être prise de manière libre, cela se résout en une discussion à plusieurs voix qui ne tendent pas à une négociation. Un des premiers exemples, la gestion de déchets (Barbier, 2004) met en exergue la nécessité de modifier les habitudes des maitres d'ouvrage et éviter que les collectivités instrumentalisent ces démarches de participation. 
2- la relation entre les temps du territoire et ceux des acteurs du territoire (décision, compréhension et propositions qui ne laissent pas une marge suffisante pour faire des études sur des temporalités longues.);

3- pour finir, à chaque débat public sur l'environnement, tenter de définir les échelles de validité de la discussion voire de la contestation (d'une part, comme pour les revendications urbaines classiques, il faut apprendre à les inscrire dans un contexte territorial de proximité, mais, en même temps, leur échelle est bien plus importante que celle des effets locaux, car le territoire de l'environnement est la planète entière).

Ce constat permet de souligner la complexité et les paradoxes à l'œuvre dans les questions territoriales (urbaines, infrastructurelles, environnementales) et leurs discours participatifs. Tout particulièrement, le caractère scalaire des débats, invoque une analyse attentive des acteurs et de leurs compétences et capacités à s'adapter. Pour surmonter les solutions rationalisantes (cadres institutionnels trop pré-définis), il faudrait parier sur la possibilité d'offrir à la participation la possibilité de s'inscrire dans un cadre complexe. L'urbain et ses environnements sont en effets des cadres complexes multiscalaires. Ainsi les innovations territoriales et sociales devraient avoir - à la fois- un caractère d'unicité, mais en même temps une possibilité de convenir à des cas comparables. Pour ce faire, il faudrait s'appuyer sur les exemples et les analyses conduits dans d'autres pays et tester leur capacité à exister sur un territoire. Il s'agirait ainsi moins de chercher des modèles (Moulaert, Delladetsina, 1997) que de tenter des processus et des procédures réellement innovants.

\section{Conclusion : implémenter l'innovation dans l'espace du territoire}

Le débat des années 1990 a été caractérisé par un tournant discursif, dont l'innovation sociale (coopération et participation des acteurs sociaux et usagers) devait constituer l'embrayage capable de réformer les politiques territoriales innovantes. Au début des années 2000, l'importance du territoire dans ses dimensions spatiales s'impose fortement, notamment à travers les lectures scalaires (Brenner, 2001). A la suite de cette « respatialisation» du discours, le territoire ne cesse de se redéfinir (Proulx, 2008 ; Vanier, 2009 ; Godet, Durance, Mousli, 2010 ; Fontan, 2011) et de définir ses périmètres de compétence (géographique, politique, culturel...). Ainsi, au participatory turn (Pestre, 2011) succède un spatial turn qui cherche à comprendre les impacts réels des innovations dans un contexte complexe (Marston, 2005). La question centrale est celle de la possibilité d'appliquer des discours délibératifs dans un territoire, ou mieux la possibilité de "donner forme » aux pratiques discursives du «bien commun » et les incarner dans des projets d'innovation territoriale. Comme le montrent les recherches de Moulaert (2006) : "L'élément décisif dans le discours de l'innovation sociale se fonde sur la possibilité de vérifier les avantages sur le territoire. En effet, l'apparition du territoire et, plus précisément, le lien entre l'innovation sociale et l'avantage, les retombées, pour le territoire légitime ces démarches comme des leviers d'action) ».

L'articulation se réalise dans une strate intermédiaire dont les formes émergent progressivement. Au lieu de définir les contenus, on se limite à observer l'«homothétie» possible entre le discours sur l'innovation, qui émerge de la société (innovation sociale) et devrait alimenter le renouvellement des institutions, et le management innovant (gestion et aménagement) des territoires. ${ }^{16}$ Une révision critique des rapports entre gestion, environnement et créativité (Florida, 2000), comme celle évoquée par Michael Mumford (2013), permet d'établir le lien possible entre la grande échelle (celle de l'innovation institutionnelle, technologique et industrielle) et les micro-innovations, à la condition de redéfinir et évaluer les impacts en fonction de leur «étendue » (breadth). Pour donner un caractère

\footnotetext{
${ }^{16} \mathrm{Il}$ faut souligner ici une distinction conceptuelle qui a manqué lors de la traduction -au sens littéral- du terme government dans la littérature sur la participation : ce terme a été rapidement traduit comme l'Etat, alors qu'il renvoie, d'une part, à la notion managériale de gestion et, d'autre part, à la gouvernance locale (Rochet, 2010). C'est au prisme de ce terme qu'il faut lire l'importance de l'échelle locale dans les théories anglo-saxonnes et canadiennes et tenter de voir comment cela peut être implémenté à l'échelle du territoire français. 
prospectif à cette lecture relationnelle, il serait aussi nécessaire d'observer comment fonctionnent les modes du transfert et, tout particulièrement, la notion de « proximité », entendue au sens géographique (spatial) et organisationnel (qui mobilise les interactions). Des études (Torre et Gilly, 2000) ont montré la force d'impact endogène des espaces sur l'innovation et tout particulièrement la relation des formes de proximité et de l'apprentissage et l'innovation (Asheim 1996 ; Lundvall, 1988). Si la proximité est bien une modalité de transfert (Boschma, 2004), il faut l'observer dans sa double face, celle spatiale et celle d'interaction (Balland, 2012), pour comprendre les caractéristiques propres à chaque contexte.

Dans les recherches sur la possibilité de lier ces termes, en France (Le Coroller, 2012), la force d'un(e) path dependency du contexte historique et, tout particulièrement, la trajectoire d'innovation propre au territoire a une influence plus marquée que dans les contextes américains. Des études lancées par le Commissariat Général à l'Egalité des Territoires ${ }^{17}$, montrent aussi que l'acteur public (collectivités, Etat, services déconcentrés ou décentralisés) manifeste son incompréhension par rapport au rôle à jouer, celui du facilitateur (mise à disposition de lieu de travail, mise en place de réseaux, mise à disposition de datas, éditorialisation des connaissances, propositions de chartes de partenariat plus que des contrats). Il semblerait donc que nous nous retrouvons dans une nouvelle phase où le participatif est devenu un «modèle d'action» (innovation sociale; design social) dont l'échelle d'application n'a pas été suffisamment définie par rapport à l'échelle des institutions chargées de le mettre en œuvre.

Confrontées à l'histoire politique de chaque pays, les politiques chargées d'entendre les revendications urbaines - innovation urbaine, innovation sociale et démarches participatives- mettent en évidence des aspects non transférables d'une culture à l'autre. Ces aspects concernent les interfaces institutionnelles qui sont spécifiques à chaque culture politique nationale et permettent de donner une forme spatiale aux discours. Ainsi, la question des échelles et leurs relations (proximités et scalabilité), peu présente dans les pratiques d'aménagement urbain, en France, freine la possibilité d'implémenter le discours de l'innovation sur le territoire et d'encastrer le social dans celui-ci.

\section{Bibliographie}

AKRich M., CAllon M., LATOUR B., « À quoi tient le succès des innovations. Premier épisode : l'art de l'intéressement», Annales des Mines, $\mathrm{n}^{\circ} 11$, juin 1988, pp. 4-17; suivi de « À quoi tient le succès des innovations. Deuxième épisode : l'art de choisir les bons porte-parole », Annales des Mines, n¹2, septembre 1988, pp. 14-29.

Alvergne C., Taulelle F, Du local à l'Europe : les nouvelles politiques d'aménagement du territoire, Paris, Presses universitaires de France, 2002.

Allain S., "Social Participation in French Water Management: Contributions to River Basin Governance and New Challenges ", in Berry K. A., Mollard E. (dir.), Social Participation in Water Governance and Management, London, Earthscan, 2010, p. 95-114.

Аміот M., Contre l'État, les sociologues. Éléments pour une histoire de la sociologie urbaine en France, 1900-1980, Paris, Éditions de l'École des hautes études en sciences sociales, 1986.

AnSELme M., Du bruit à la parole. La scène politique des cités, La Tour d'Aigues, Éditions de l'Aube, 2000.

ARAB N., VIVANT E., «L'innovation de méthodes en urbanisme : freins et leviers d'une entreprise incertaine », CRAUP, Cahiers de la recherche architecturale urbaine et paysagère , 1, 2018 : http://journals.openedition.org/craup/324; DOI : $10.4000 /$ craup.324

ARAB, N., L'urbanisme et l'innovation, mémoire d'HDR, vol. 2. Université Paris-Est, 2014.

ARNSTEIN S., « A Ladder of Citizen Participation », JAIP, 35 (4), 1969, p. 216-224.

ASCHER F., Les nouveaux principes de l'urbanisme, La Tour d'Aigues, éditions de l'Aube, 2010.

\footnotetext{
${ }^{17}$ Ces études intéressantes sont peu utilisées dans les milieux professionnels de l'aménagement probablement en raison de l'absence des acteurs institutionnels à leur mise en œuvre ; les acteurs impliqués dans le débat sont peu représentatifs : issus du monde associatif lié à l'innovation ou à des structures publiques accompagnant l'innovation, ils sont bien repérés et deviennent des interlocuteurs presque officiels. 
ASHEIM B., «Industrial districts as 'learning regions': a condition for prosperity». European Planning Studies, 1996, 4, 4, p.379- 400 .

AUDIER, S., La société écologique et ses ennemis, Paris, La Découverte, 2017.

BAdré M., DE Fleurieu A., Juffé M., La gouvernance concertée, Paris, Conseil Général de l'Environnement et du Développement Durable, Rapport $\mathrm{n}^{\circ}$ 006766-00, 2010, http://portail.documentation.developpementdurable.gouv.fr/documents/cgedd/006766-00_rapport.pdf.

BALLAN E., « Démocratie et environnement, un mariage arrangé », Territoires, 516, 2011, p. 28-29.

BALLAND P.A. " Proximity and the evolution of collaboration networks: evidence from research and development projects within the Global Navigation Satellite System (GNSS) industry», Regional Studies 46, 2012, 741-756.

BAqUe M. H., GaUthieR M., « Participation, Urbanisme et Etudes Urbaines. Quatre décennies d'expériences et de débat depuis "A ladder of citizen participation" de S. R. Arnstein », Participations, 2011/1, pp. 36-66.

BARATTUCCI C., Urbanisations dispersées : interprétations-actions, France et Italie, 1950-2000, Rennes, PUR, 2006

Barbier R., RiauX J., BarReteau O., « Science réglementaire et démocratie technique», Natures Sciences Sociétés, 18 (1), 2010, p. 14-23.

BARBIER R., LARRUE C., «Démocratie environnementale et territoires, un bilan d'étape », Participations, 2011/1, p. 67104.

BECK U., La société du risque, Aubier, 2001 (1991).

Belanger P., LeVeSQue B., « La théorie de la régulation, du rapport salarial au rapport de consommation. Un point de vue sociologique », Cahiers de recherche sociologique, no 17, 1992, pp. 17-51.

BERQUe A., Écoumène. Introduction à l'étude des milieux humains, Paris, Belin, 2000.

Beuret J.-E., Pennanguer S., TARTARIN F., « D’une scène à l'autre, la concertation comme itinéraire », Natures Sciences Sociétés, 14 (1), 2006, p. 30-42.

BLANC N., Vers une esthétique environnementale. Paris, Quae, 2008.

Blanchot F., PAdioleau J.-G., « Une économie politique du travail journalistique », Hermès, La Revue 2003/1 (n 35), p. 63-71.

BlatriX C., La "démocratie participative", de mai 1968 aux mobilisations anti-TGV. Processus de consolidation d'institutions sociales émergentes, Thèse de science politique, Université Paris 1- Panthéon Sorbonne, 2000

Boren T., Craig Young, " Getting creative with the "Creative City"? Towards new perspectives on creativity in urban policy », International Journal of Urban and Regional Research, 37 (5), 2013, pp. 799-815.

BoschMA R.,«Proximité et innovation ». Économie rurale, 280, 2004, pp. 8-24; doi : 10.3406/ecoru.2004.5469

Boudon P., De l'architecture à l'épistémologie : la Question de l'échelle, Paris, Puf, coll. « Nouvelle Encyclopédie Diderot », 1990.

Bourg D., La Pensée écologique. Une anthologie, Paris, Puf, coll. «L'écologie en questions », 2014.

Bourg D., Dictionnaire de la pensée écologique, Paris, Puf, 2015.

BREUX S., COLLIN, GINGRAS, Représenter l'urbain : apports et méthodes, Presses de l'Université Laval, 2014.

BUSQUET G., Idéologie urbaine et pensée politique dans la France de la période 1958-1981. Thèse de doctorat en urbanisme et aménagement, Institut d'urbanisme de Paris, Université Paris 12, 2007.

BRENNER N., « The limits to scale », Progress in Human Geography 25,4 (2001) pp. 591-614.

BRundtland G. H., Our Common Future, Report for the World Commission for Environment and Development, 1987.

CAlthorpe P., The next American metropolis, Princeton Architectural Press, 1993.

CALthorPe P., Fulton W., The Regional City, Islandpress, 2001.

CALlON M., «Le travail de conception en architecture », Cahiers de la recherche architecturale, $\mathrm{n}^{\circ}$ 37, 1996, pp. 25-35.

CANs R., Petite histoire du mouvement écolo en France, Delachaux et Niestle, 2006.

CARDON D., GRANJON F., Médiactivistes, Presses de la Fondation Nationale des Sciences politiques, 2013.

CARo P., DARD, O., Daumas J. P., La politique d'aménagement du territoire: Racine, logiques et résultats, Presses Universitaires de Rennes, 2002. 
CARON F., La dynamique de l'innovation, Paris, Gallimard, 2010.

ChiAPPero F., Du Collectif Etc aux «collectifs d'architectes ». Thèse sous la direction de Stéphane Hanrot, soutenue à l’Université Aix-Marseille, novembre 2017.

CHOAY F. L'urbanisme utopie et réalité. Paris, La découverte, 1979

ChOAY F., MERLin P., Dictionnaire de l'urbanisme et de l'aménagement, PUF, « Quadrige dicos poche », 2015 (1988).

CLAVAL P., «Les interprétations fonctionnalistes et les interprétations symboliques de la ville», Cybergeo : European Journal of Geography [Online], Topics, document 81, Online since 10 March 1999, connection on 13 April 2018. URL : http://journals.openedition.org/cybergeo/1069; DOI : 10.4000/cybergeo.1069

ClAeYS-MeKDADE C., " La participation environnementale à la française : le citoyen, l'État... et le sociologue », VertigO, 7 (3), 2006, http://vertigo.revues.org/8446.

CONAN M., Concevoir un projet d'architecture, Paris, L’Harmattan, 1990.

DAMANPOUR, F., "Organizational Innovation: A meta-analysis of the effects of determinants and moderations », Academy of Management Journal 34, 555-590.

Deleage J.P., Une histoire de l'écologie, La découverte, 1991.

DESJEUX D., L'empreinte anthropologique du monde. Méthode inductive illustrée. Peter Lang, 2018.

DESLAURIERS J.P., Cadre de référence pour l'appropriation du pouvoir d'agir personnel et collectif, Centre d'étude et de recherche en intervention sociale, Université du Québec en Outaouais, 2007.

ELLIN, N., Postmodern Urbanism. Oxford, Blackwell, 1996.

FISHMAN, R., «The Anti-Planners: the Contemporary Revolt Against Planning and Its Significance for Planning Theory», G.E. Cherry, Shaping an Urban World. Londres, Mansell, pp. 243-252.

FLEURY A., WUEST L., «Vers de nouveaux modes de production des espaces publics à Paris? Réflexions à partir du projet "Réinventons nos places"», Métropolitiques, 18 mars 2016.URL: http://www.metropolitiques.eu/Vers-de-nouveauxmodes-de.html

FLIPO F. Le développement durable, Rosny-sous-Bois, Thèmes \& débats, Bréal, 2007.

FLORIDA R., The Rise of the Creative Class : And how it is Transforming Work, Leisure, Community and Everyday Life, Basic Books, 2003.

Fontan J.-M., Hamel P., Morin R., Shragge E., « Le développement local dans un contexte métropolitain. La démocratie en quête d'un nouveau modèle », Politique et Sociétés, 25 (1), 2006, p. 99-127.

Forest J., HAMDOUCH A., Quand l'innovation fait la ville durable, Presses polytechniques et universitaires Romandes, Lausanne, 2015.

FORESTER J., Planning in the face of Power, Berkeley University Press, 1989.

FORESTER J., «Beyond 'Participation': From Risk Management to Processes of Dialogue, Debate, and Negotiation», Building Safer Communities. Risk Governance, Spatial Planning, and Responses to Natural Hazards, 2009.

FRENKEN K.,Understanding product innovation using complex systems theory. University of Amsterdam - University of Grenoble, Ph.D. Thesis, 2001.

GAGLIO G., Sociologie de l'innovation. Paris, PUF (Que sais-je), 2011.

GARNIER J.-P., 2001, «Urbaniser pour dépolitiser: la rhétorique du spatialisme et du localisme », http://infokiosques.net/IMG/garnier_A4.pdf.

GHORRA-GOBIN C., La métropolisation en question, Paris, PUF, 2015.

GiBSON-GrahAM, JK, « An ethics of the local ». Rethinking Marxism 15 (1), 2003, 49-74.

Godet M., Durance P., Mousli M., Créativité et innovation dans les territoires. Rapport du Conseil d'Analyse Économique, Direction de l'information légale et administrative, La Documentation française, Paris, 2010.

Godin B., Innovation and Science: When Science Had Nothing to Do with Innovation, and Vice-Versa. Project on the Intellectual History of Innovation Working Paper No. 16, 2014.

GotTdiener M., The New Urban Sociology, Mcgraw-Hill College, 1993.

Gourgues G., RUI S., TOPÇU S., « Gouvernementalité et participation. Lectures critiques », Participations, 2013/2 (N 6), p. 5-33. DOI : 10.3917/parti.006.0005. URL : https://www.cairn.info/revue-participations-2013-2-page-5.htm 
GRAS A., Grandeur et dépendance. Sociologie des macrosystémes techniques, PUF, Paris, 1993.

HAMEL P., « Les pratiques planificatrices dans le contexte actuel. Comment interpréter l'appel à la participation ?», Revue internationale d'action communautaire, 15 (55), 1986, p. 65-76.

HARDIN G., « The Tragedy of the Commons», Science, 13 décembre 1968, vol. 162. n ${ }^{\circ} 3859$, p. 1243-1248.

HARVEY D., Social justice and the city. Oxford: B. Blackwell, 1973.

HARVEY D., Spaces of Hope. Los Angeles: University of California Press, 2000.

HATZFELD H., «Municipalités socialistes et associations. Roubaix : le conflit de l'Alma-Gare », Revue française de science politique, 36, n³, 1986. pp. 374-392.

HeAley P., Collaborative Planning. Shaping Places in Fragmented Societies, Vancouver, UBC Press, 1997.

HEMMENS J.C., STIFFEL B., « Sources for the Renewal of Planning Theory », APA Journal, 46: 341-345.

JACOBS J., Déclin et survie des grandes villes américaines, Marseille, Parenthèses, 2012 (1961).

JOSEPH I., «L'espace public comme lieu de l'action ». Les Annales de la recherche urbaine, N57-58, 1992, pp. 211-217.

KASLER D., Max Weber: sa vie, son oeuvre, son influence. Paris, Fayard, 1996.

KLEIN, J., HARRISSON, D., L'innovation sociale : Émergence et effets sur la transformation des sociétés, Québec, PUQ

KunZMAnN K., « Creativity in Planning: a Fuzzy Concept? », disP - The Planning Review, n 162, 2005, pp. 5-13.

MARSTON S., JONES J.P., WOODWARD K., « Human geography without scale », Trans Inst Br Geogr, 30, 2005, 16- 432.

MERMET L., BERLAN-DARQUÉ M., Environnement : décider autrement, Paris, L’Harmattan, 2009.

LE Coroller C., «Mots et sens du territoire dans les démarches d'innovation sociale en Basse Normandie », Géographie Economie Société, Lavoisier, 2012, 14 (3), pp.287-302.

LE GALES P. «Du gouvernement des villes à la gouvernance urbaine », Revue française de science politique, 1995/1 (Vol. 45), p. 57-95. URL : https://www.cairn.info/revue-francaise-de-science-politique-1995-1-page-57.htm

LEFEBVRE H., Le droit à la ville, Paris, Anthropos, 1968.

LEONTIDOU L., «Alternatives to Modernism in (Southern) UrbanTheory: Exploring In-Between Spaces », International Journal of Urban and Régional Research, 1996, 20 (2): 178-195.

Leroy P., Van Tatenhove J.P.M., «Environment and Participation», Driessen P.P.J., Glasbergen P., Greening Society. Environment \& Policy, vol 33. Springer, Dordrecht, 2002.

LEVY J., LuSSAUlt J., Logiques de l'espace, esprit des lieux. Géographies à Cerisy. Paris, Belin, « Mappemonde », 2000.

LEVY J., Réinventer la France, Paris, Fayard, 2013.

LINOSSIER R., « Le conseil en stratégies et projets urbains : un marché atypique », Politiques et management public [En ligne], Vol 29/1 | 2012 : http://journals.openedition.org/pmp/4733

LUNDVALL B-A. « Explaining interfirm cooperation and innovation. Limits of he transaction-cost approach», G. Grabher, The embedded firm. On the socioeconomics of industrial network, Routledge, London, 1993, p. 52- 64.

LuSSAult M., L'avènement du Monde. Essai sur l'habitation humaine de la Terre, Seuil, coll. « La couleur des idées », 2013.

LUSSAUlT M., L’homme spatial, Paris, Seuil, 2007.

LuSSAUlt M, Hyperlieux, Paris, Seuil, 2017.

MAGNAGHI A., Le projet local, Mardaga, 2003 (2000).

Magnaghi A., La Biorégion urbaine, petit traité sur le territoire bien commun, Paris, Eterotopia France, 2014

Mansbridge J., Bohman J., Chambers S., Estlund D., Føllesdal A., Fung A., Lafont C., Manin B., Martí J.-L., " The Place of Self Interest and the Role of Power in Deliberative Democracy », The Journal of Political Philosophy, 18 (1), 2010, p. 64-100.

MARSTON S., JONES J.P., WOODWARD K., «Human geography without scale», https:// doi.org/10.1111/j.1475$\underline{5661.2005 .00180}$

Merlin P., L'aménagement du territoire en France, Paris, la Documentation française, coll. «Les études de la Documentation française », 2007. 
MiLroy B., « Into Postmodern Weightlessness ». Journal of Planning Education and Research, 10 (3): 181-187, 1991

MOLLARD E. Analyse politique du conflit environnemental. [En ligne], 2012.

MORISSET L., NOPPEN L., «Le patrimoine immatériel: une arme à tranchants multiples», Téoros 24-1|2005: http://journals.openedition.org/teoros/1500

Mouffe C., «Democratic Citizenship and The Political Community», Dimensions of Radical Democracy, Pluralism, Citizenship, Community, Verso, Londres, 1992.

MoufFe C., The democratic paradox, London, Verso 2000 (traduit en 2016).

Moulaert, F., Delladetsima, P., Delvainquière, J.C., Demazière, Ch., Leontidou, L., Local Development Strategies in Economically Disintegrated Areas: A Pro-Active Strategy against Poverty in the European Community. Reports for the EC, DG Research. IFRESI-CNRS, Lille, 1992-1994.

MumForD, M.D., « Social Innovation: Ten Cases from Benjamin Franklin ». Creativity Research Journal 14 (2), 2002, 253-266.

Mumford M.D., MOERTL P., «Cases of Social Innovation: Lessons From Two Innovations in the 20th Century », Creativity Research Journal, 15:2-3, 2011, 261-266.

NusSBAUMER, J., Le rôle de la culture et des institutions dans les débats sur le développement local : la contribution de l'École Historique Allemande. Thèse de doctorat en sciences économiques. Université de Lille 1, 2002.

Nussbaumer J., Hillier J., MoulaerT F. « Trois essais sur le rôle de l'innovation sociale dans le développement territorial », Géographie, Economie et Société, 2006, 6, 129-152.

NoOTEBoOM B., Learning and Innovation in Organisations and Economics, Oxford, Oxford University Press, 2000.

Nyssen, Defourny J., Hulgard L., Pestoff V., «Social Entreprise, Social Entrepreneurship, Social Economy, Solidarity Economy : An EMES Reader on the "SE Field" », EMES European Research Network, 2010.

OSTROM E., La gouvernance des biens communs : pour une nouvelle approche des ressources naturelles [« Governing the Commons: The Evolution of Institutions for Collective Action »], Commission Université Palais, 2010.

OZDIRLIK B., TERRIN J., La conception en question. La place des usagers dans les processus de projet, La Tour d'Aigues, Éditions de l'Aube, 2015.

OURAL A., L'innovation au pouvoir, Rapport pour le SGMAP-CRED, 2015.

PAdioleau J.-G., « L'action publique. Du substantialisme au pragmatisme », Techniques, Territoires, Sociétés, 22/23, 2000, pp. 89-95.

PERULLi P., Visioni di città. Le forme del mondo spaziale, Piccola Biblioteca Einaudi, Torino, 2009.

PESTRE D., « Des sciences, des techniques et de l'ordre démocratique et participatif », Participations 2011/1, pp. 210-238

PICON A., Digital culture in architecture : An Introduction, Birkhäuser, 2010.

PINSON G., « Projets de ville et gouvernance urbaine. Pluralisation des espaces politiques et recomposition d'une capacité d'action collective dans les villes européennes », Revue française de science politique, 56 (4), 2006, p. 619-651.

PInSON, G., Gouverner la ville par projet, Paris, Presses de Sciences Po, 2009.

PROST R, Pratiques de projet en architecture. Le tournant silencieux, InFolio, 2014.

ProulX M.-U., " 40 ans de planification territoriale au Québec », in Gauthier M., Gariépy M., Trépanier M. O., Renouveler l'aménagement et l'urbanisme, Montréal, Presses de l'Université de Montréal, 2008, p. 23-54.

RAPPAPORT J., ROBERT H., Studies in Empowerment: Steps Toward Understanding and Action, Routledge, 1984.

RAINEAU L., «Innover autrement : la technique face à la crise environnementale », Habitat et transition énergétique, sous la direction de François Rochon, Paris, L'Harmattan, 2014, pp. 49-69.

ReY-VALETTE H., MATHE S., «L'évaluation de la gouvernance territoriale. Enjeux et propositions méthodologiques », Revue d'Économie Régionale \& Urbaine, 2012/5 (décembre), p. 783-804 : 10.3917/reru.125.0783.

Rosanvallon P., La légitimité démocratique : Impartialité, réflexivité, proximité, Paris, Seuil, 2008.

RoBic M.-C., Du milieu à l'environnement. Pratiques et représentations du rapport homme/nature depuis la Renaissance, Economica, 1992.

Rochet C., « Pas de philosophie, SVP, nous sommes des managers », Revue Internationale des Sciences Administratives, 2010, pp. 302-335. 
RUMPALA Y., "Le "développement durable" appelle-t-il davantage de démocratie? Quand le "développement durable" rencontre la "gouvernance"... »,VertigO, 8 (2), 2008 : http://vertigo.revues.org/4996

SAINT-PIERRE de C., La fabrication plurielle de la ville : décideurs et citadins à Cergy-Pontoise, 1990-2000, Paris, Créaphis, 2002.

SAlAt S., LABBE F., NOWAKI C. Les Villes et les formes. Sur l'urbanisme durable, Hermann, Paris, 2011.

SCHERRER F., « Le contrepoint des études urbaines et de l'urbanisme. Ou comment se détacher de l'évidence de leur utilité sociale », Tracés, 3 (HS-10), 2010, p. 187-195.

SHIVA, V., The Violence of the Green Revolution - Third World Agriculture, Ecology and Politics, Paperback, 1991.

SCHÖN D., « The architectural studio as an exemplar for reflection-in-action », Education, n³8, 1984, pp. 2-9.

SoJa E., Postmetropolis: Critical Studies of Cities and Regions, Wiley-Blackwell, 2000.

SOMMER R., Social Design , Prentice-Hall, 1983.

SOMMER R., Milieux et modes de vie, Infolio, 2003.

TABARIÉS M., «Les apports du GREMI à l'analyse territoriale de l'innovation ou 20 ans de recherche sur les milieux innovateurs », Cahiers de la Maison des Sciences Economiques, 18, 2005.

TAPIE G., Du politique à l'oeuvre : système et acteurs des grands projets urbains et architecturaux (Bilbao, Bordeaux, Bercy, San Sebastian), La Tour d'Aigues, Aube, 2000.

TsIOMIS Y., Echelles et temporalités des projets urbains (2002-2007), PUCA-Plan Urbanisme Construction Architecture, JMP, 2007.

TIRYAKIAN E. A., « Dialectics of Modernity: Reenchantment and Dedifferentiationas Counterprocesses», H. HAFERKAMP ET N.J. SMELSER, Social Change and Modernity.Berkeley, University of California Press, 1992, pp. 78-94.

Topalov C., "Thirty Years of Urban Sociology. A French Viewpoint », Metropolitics, 30 October 2015. URL: http://www.metropolitiques.eu/Thirty-Years-of-Urban-Sociology.html.

TORRE A., GILly J-P., « On the analytical dimension of proximity dynamics ». Regional Studies, 2000, 34, 2, p. 169-180.

TORRE A., ZUINDEAU B. , « Proximity economics and environment: assessment and prospects », Journal of Environmental Planning and Management 52, 1-24, 2009.

VANIER M., Territoires, territorialité, territorialisation. Controverses et perspectives, Rennes, Presses Universitaires de Rennes, 2009.

Vinck D., JeAnteT A., « Mediating and Commissioning Objects in the Sociotechnical Process of Product Design: a conceptual approach», MAClEAN D., SAVIOTTI P., VINCK D., Designs, Networks and Strategies, vol. 2, COST A3 Social Sciences. Bruxelles: EC Directorate General Science R\&D, 1995, p. 111-129.

VINCK D., Ingénieurs au quotidien. Ethnographie de l'activité de conception et d'innovation. Grenoble, PUG, 1999.

WILliams, R.H., European Union Spatial Policy and Planning. Liverpool, Paul Chapman, 1996.

ZEVI B., Dialectes architecturaux = Dialetti architettonici . Paris, Ed. du Linteau, 2016 (1996). 